\title{
8
}
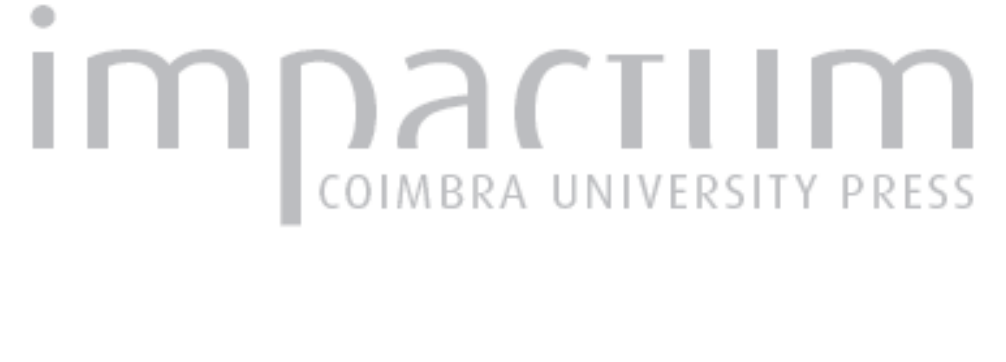

\section{Análise temporal (2002-2014) da expansão da espécie de samambaia dicranopteris flexuosa no Vale do Pati (Brasil) utilizando técnicas de classificação de imagens de satélite}

$\begin{array}{ll}\text { Autor(es): } & \begin{array}{l}\text { Leite, Cândida Caroline Souza de Santana; Santos, Sarah Moura Batista } \\ \text { dos; Rocha, Washington de Jesus Sant'anna da Franca; Vasconcelos, } \\ \text { Rodrigo Nogueira }\end{array} \\ \text { Publicado por: } & \begin{array}{l}\text { Associação Portuguesa de Riscos, Prevenção e Segurança; Imprensa } \\ \text { da Universidade de Coimbra }\end{array} \\ \begin{array}{ll}\text { URL } \\ \text { persistente: }\end{array} & \text { URI:http://hdl.handle.net/10316.2/41230 } \\ \text { DOI: } & \text { DOI:https://doi.org/10.14195/1647-7723_24_10 } \\ \text { Accessed : } & \text { 26-Apr-2023 03:19:23 }\end{array}$

A navegação consulta e descarregamento dos títulos inseridos nas Bibliotecas Digitais UC Digitalis, UC Pombalina e UC Impactum, pressupõem a aceitação plena e sem reservas dos Termos e Condições de Uso destas Bibliotecas Digitais, disponíveis em https://digitalis.uc.pt/pt-pt/termos.

Conforme exposto nos referidos Termos e Condições de Uso, o descarregamento de títulos de acesso restrito requer uma licença válida de autorização devendo o utilizador aceder ao(s) documento(s) a partir de um endereço de IP da instituição detentora da supramencionada licença.

Ao utilizador é apenas permitido o descarregamento para uso pessoal, pelo que o emprego do(s) título(s) descarregado(s) para outro fim, designadamente comercial, carece de autorização do respetivo autor ou editor da obra.

Na medida em que todas as obras da UC Digitalis se encontram protegidas pelo Código do Direito de Autor e Direitos Conexos e demais legislação aplicável, toda a cópia, parcial ou total, deste documento, nos casos em que é legalmente admitida, deverá conter ou fazer-se acompanhar por este aviso. 


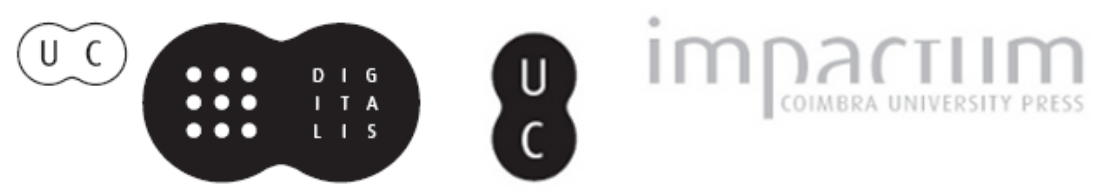

impactum.uc.pt digitalis.uc.pt 


\section{MULTIDISCIPLINARIDADE NA ANÁLISE DAS MANIFESTAÇÕES DE RISCO}

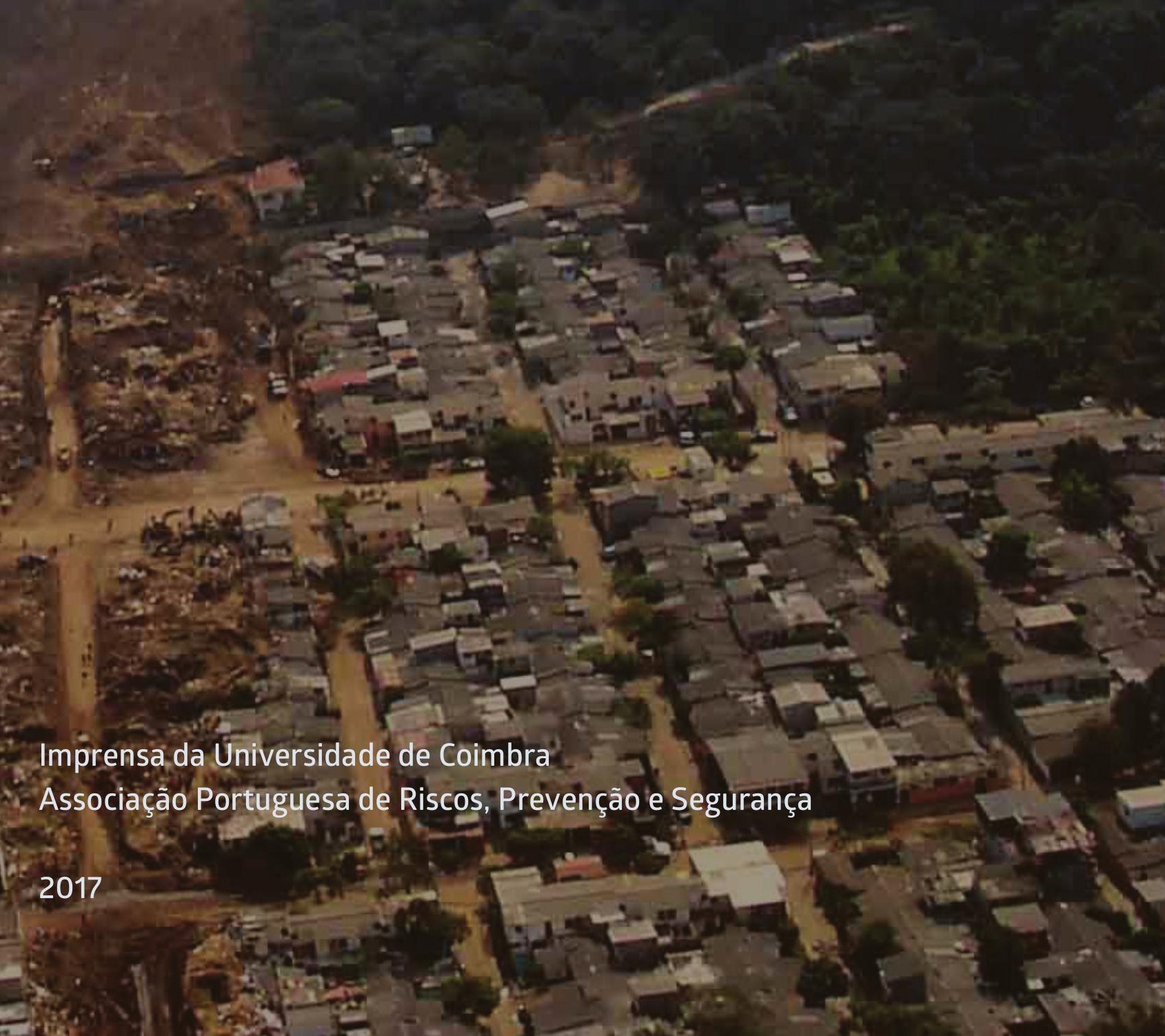




\title{
ANÁLISE TEMPORAL (2002-2014) DA EXPANSÃO DA ESPÉCIE DE SAMAMBAIA DICRANOPTERIS FLEXUOSA NO VALE DO PATI (BRASIL) UTILIZANDO TÉCNICAS DE CLASSIFICAÇÃO DE IMAGENS DE SATÉLITE*
}

\begin{abstract}
TEMPORAL ANALYSIS (2002-2014) OF THE EXPANSION OF FERN SPECIES DICRANOPTERIS FLEXUOSA IN PATI VALLEY (BRAZIL) USING SATELLITE IMAGES CLASSIFICATION TECHNIQUES
\end{abstract}

\author{
Cândida Caroline Souza de Santana Leite \\ Departamento de Ciências Humanas e Filosofia \\ Universidade Estadual de Feira de Santana \\ candidaleite.geo@gmail.com \\ Sarah Moura Batista dos Santos \\ Universidade Estadual de Feira de Santanal \\ saamoura@gmail.com
}

\author{
Washington de Jesus Sant'anna da Franca Rocha \\ Universidade Estadual de Feira de Santana \\ wrocha@uefs.br \\ Rodrigo Nogueira Vasconcelos \\ Universidade Estadual de Feira de Santa \\ rnvuefsppgm@gmail.com
}

\begin{abstract}
RESUMO
Afim de analisar as áreas ocupadas pela espécie de samambaia invasora Dicranopteris flexuosa no período entre 2002 e 2014, no Vale do Pati (Bahia-Brasil), foram utilizadas técnicas de classificação de imagens de satélite multiespectrais a partir dos algoritmos de classificação supervisionada Redes Neurais e Máxima Verossimilhança. Os resultados apontam que a espécie tende a se expandir rapidamente, impedindo a regeneração da flora nativa. 0 algoritmo que apresentou maior confiabilidade para tal foi o Redes Neurais.
\end{abstract}

Palavras-chave: Redes neurais, máxima verossimilhança, geotecnologias, espécie invasora.

\section{ABSTRACT}

In order to analyze the areas occupied by the invasive fern species Dicranopteris flexuosa between 2002 and 2014 in the Pati Valley (Brazil) multispectral satellite image classification techniques were used from the supervised classification algorithms Neural Networks and Maximum Likelihood. The results show that the species tends to spread rapidly, blocking the regeneration of native flora. The algorithm with the highest reliability for this was the Neural Networks.

Keywords: Neural networks, maximum likelihood, geotechnology, invasive species.

\section{RESUMEN}

Análisis temporal (2002-2014) de la expansión de la especie de helecho invasiva Dicranopteris flexuosa en el valle de Pati (Brasil) utilizando técnicas de clasificación de imágenes de satélite - Con el objetivo de analizar las áreas ocupadas por la especie Dicranopteris Flexuosa en un período de tiempo entre 2002 y 2014, en el Valle de Pati (Bahía-Brasil), fue necesario utilizar técnicas de clasificación de imágenes de satélite multi-espectrales a partir de los algoritmos de clasificación supervisada Redes Neuronales y Máxima Verosimilitud. Los resultados obtenidos demostraron que la especie tiende a expandirse rápidamente, impidiendo la regeneración de la flora nativa. El algoritmo que presentó una mayor confiabilidad en sus resultados fue el basado en las Redes Neuronales.

Palabras clave: Redes neuronales, máxima verosimilitud, geo-tecnologías, especie invasora.

\section{RESUMÉ}

Analyse temporelle (2002-2014) de l'expansion de l'espèce de fougère envahissante Dicranopteris flexuosa dans la vallée du Pati (Brésil) à partir d'une technique declassification des images satellite - Afin d'analyser les zones occupées par l'espèce Dicranopteris flexuosa entre 2002 et 2014 dans la vallée du Pati (Bahia-Brésil) nous avons utilisé des techniques de classification d'images de satellite multispectrales à partir des algorithmes de classification supervisée de réseaux de neurones et maximum de vraisemblance. Les résultats montrent que l'espèce a tendance à se développer rapidement, empêchant la régénération de la flore indigène. L'algorithme avec la plus grande fiabilité pour ce but fut le Réseaux de neurones.

Mots-clé: Neural networks, maximum likelihood, geotechnology, invasive species.

* O texto deste artigo foi submetido em 30-09-2015, sujeito a revisão por pares a 09-01-2016 e aceite para publicação em 05-04-2016.

Este artigo é parte integrante da Revista Territorium, $n .^{\circ} 24,2017,{ }^{\circ} \mathrm{RIscos}, \mathrm{ISSN}$ : 0872-8941. 


\section{Introdução}

Com a criação do Parque Nacional da Chapada Diamantina (PNCD) em 1985, a comunidade que habitava o Vale do Pati, desde meados de 1844, modificou drasticamente suas formas de uso e ocupação da terra (V. Borges e J. Lobão, 2014). A história socioambiental e econômica daquele lugar sempre foi fortemente ligada à agricultura, a exemplo do cultivo do café, e ao uso de recursos naturais disponíveis, principalmente a exploração de diamante (V. Borges e J. Lobão, 2014). Contudo, após a década de 1960, estas atividades apresentaram grande declínio, levando a vegetação nativa a se recompor até tornar-se floresta em estágio médio e até avançado de regeneração (MMA, 2007).

Em estudos em campo realizados pela equipe gestora do PNCD foi observado que extensas áreas do Vale do Pati, sobretudo em locais úmidos e que sofreram antropização, foram ocupadas pela samambaia Dicranopteris flexuosa (fig. 1), espécie invasora (C. Gonçalves, 2013), e que se prolifera rapidamente, podendo causar grande desequilíbrio na fauna e flora nativa (N. Hojo-Souza et al., 2010). Ao contrário do que muito se pensa em relação, as samambaias não são restritas a ambientes úmidos, pois são plantas cosmopolitas, podendo ocorrer em diversos tipos de ambientes, contudo, têm a característica de ocorrerem principalmente em locais úmidos (F. R. Nonato, 2005).

A reprodução da $D$. flexuosa, representada na fig. 1, se dá por esporos, facilmente transportados pelo vento, bem como pela fauna local (R. M.Tryon e A. F. Tryon, 1982). Esta espécie encontra condições favoráveis de expansão em superfícies expostas e desnudas, constantemente desmatados ou queimadas, sobre solos fortemente degradados ou compactados. Uma vez instaladas, elas recorrem à multiplicação vegetativa para a exploração espacial do local (M. Queiroz, 1994) e, além de ameaçar a vegetação local com suas substâncias químicas, a

D. Flexuosa compete ativamente com esta vegetação e também impede o desenvolvimento da mesma, sombreando o terreno invadido, dificultando as espécies, que dependem de luz solar, de se instalarem, de forma a comprometer o processo natural de crescimento do vegetal (D. R. M. Lehmann 2008).

Dallman, 1998; Lambers et al., 2010; Olde Venterink, 2011 in J. L. Funk (2013), observaram que plantas com carácter invasor conseguem se adaptar e expandir mesmo em ambientes de baixos recursos como as áreas degradadas do Vale do Pati, ocupadas pela espécie $D$. flexuosa. Ambientes de baixo recursos são os quais apresentam poucos nutrientes, pouca água e baixa luminosidade. Observou-se ainda, que nestas condições há alta competividade entre as espécies invasoras e as espécies nativas (Dallman, 1998; Lambers et al., 2010; Olde Venterink, 2011 in J. L. Funk, 2013).

Este artigo descreve o padrão de distribuição geográfica dessa espécie no Vale do Pati, de forma a contribuir na compreensão da dinâmica de ocupação das samambaias no Vale do Pati (fig. 2).

O uso de técnicas de Sensoriamento Remoto destaca-se no estudo das diversas coberturas da vegetação com ênfase para as áreas agrícolas, florestas, vegetação urbana, vegetações pantanosas e pastagens (J. R. Jensen, 2007).

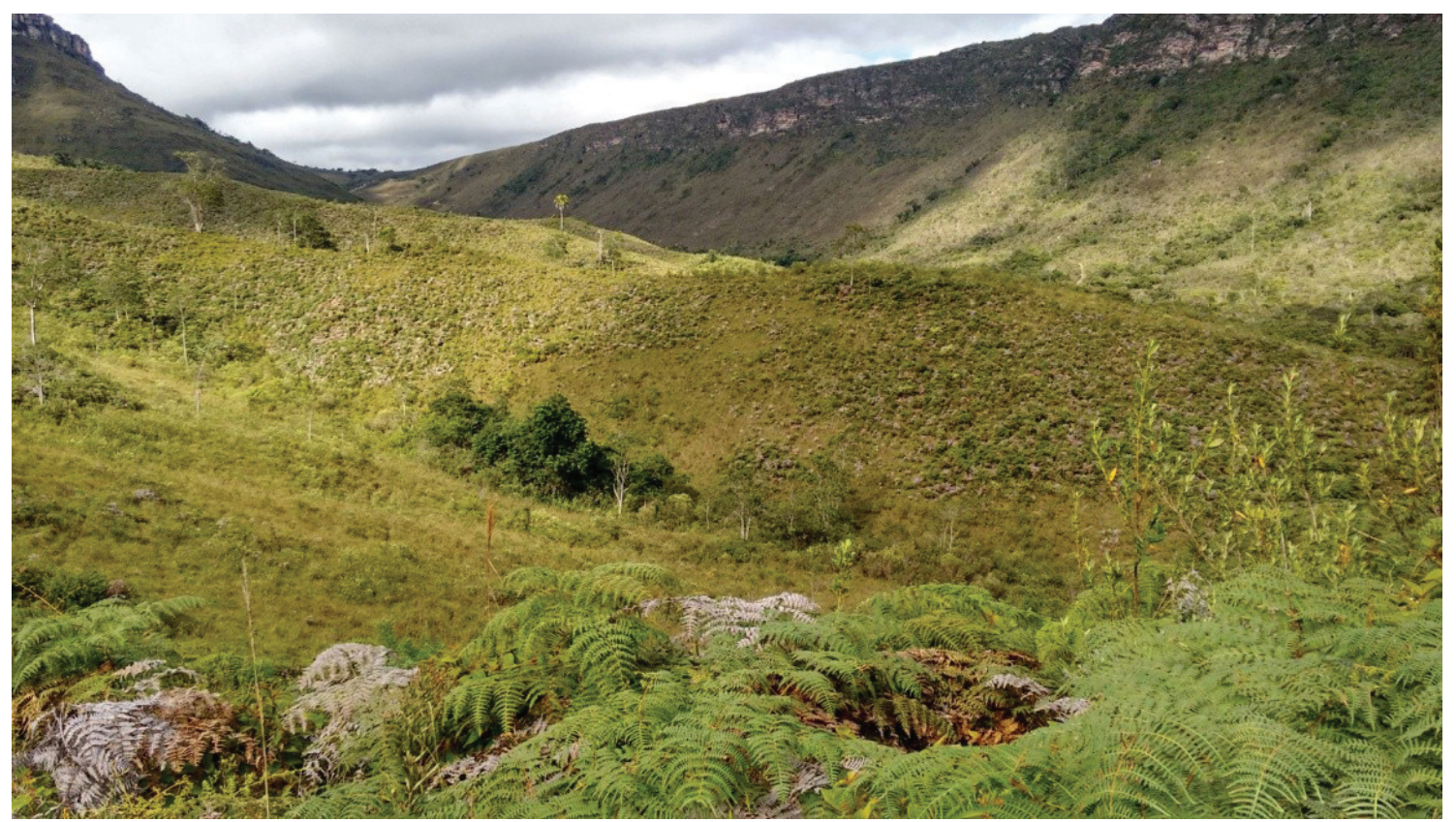

Fig. 1 - Vista da espécie Dicranopteris flexuosa na paisagem do Vale do Pati .

Fig. 1 - Dicranopteris flexuosa species view in the Pati Valley landscape. 
Um dos principais objetivos do Sensoriamento Remoto é a extração de dados e informações em imagens de satélite, onde sobressaem as técnicas de classificação de imagem (P. R. Meneses e E. E. Sano, 2012). Os algoritmos de classificação de imagens de satélite são agrupados em supervisionados e não supervisionados.

Segundo P. R. Meneses e E. E. Sano (2012), a classificação supervisionada de imagens de satélite demanda conhecimentos prévios das classes de alvos, para que seja possível classificar a imagem de acordo com classes de interesse pré-definidas. Para esta pesquisa foram utilizados os algoritmos Redes Neurais e Máxima Verossimilhança, com intuito de testar e comparar os métodos, obtendo assim um resultado mais eficaz.

O presente estudo teve por objetivo analisar a proliferação da espécie de samambaia invasora Dicranopteris flexuosa no Vale do Pati, ao longo dos anos de 2002 a 2014, com auxílio de técnicas de Sensoriamento Remoto, comparando algoritmos de classificação de imagens de satélite como Redes Neurais e Máxima Verossimilhança, mapeando as classes de uso e cobertura da terra no Vale.

\section{Materiais e métodos}

\section{Caracterização da área}

O Vale do Pati está situado no interior do Parque Nacional da Chapada Diamantina, Bahia-Brasil, que por sua vez encontra-se em um planalto conhecido como Serra do Sincorá. Tal planalto faz parte de um conjunto de relevos serranos de sentido Norte-Sul, denominado Chapada
Diamantina (R. V. Cezar, 2011). O Vale está localizado entre os municípios de Andaraí e Mucugê, inserido no grupo da Serra do Espinhaço, o qual consiste em um conjunto de terras elevadas e de topo aplainado que se estende da região central da Bahia até o norte de Minas Gerais. Por conta, principalmente, desse relevo, a Serra do Sincorá apresenta um clima do tipo tropical semi-úmido, em contraste com o clima característico do seu entorno que é o semi-árido (CPRM, 1994 in R. V. Cezar, 2011). De acordo com R. V. Cezar (2011) essa diferença de clima se dá ao fato da Chapada Diamantina agir como uma barreira, impedindo que as nuvens vindas do Oceano Atlântico atravessem as serras, causando chuvas orográficas no lado leste e deixando o lado oeste mais seco. Segundo a classificação de Köppen (1923), o clima na Chapada Diamantina é mesotérmico, o qual apresenta temperaturas mais amenas do que nas regiões circundantes (F. A. Juncá, L. Funch e W. Rocha, 2005). Além da floresta estacional e das samambaias, o Vale do Pati é constituído ainda pelos "gerais", superfícies semi-aplainadas recobertas por vegetação herbácea (G. De F. Seabra,1998) e batizadas por toponímias locais, a exemplo dos "gerais" do Vieira e o do Rio Preto.

O substrato geológico da região foi descrito por A. J. Pedreira (1997), que atribuiu às rochas encontradas nos afloramentos da Chapada Diamantina idades meso e neoproterozóicas, depositadas em um intervalo geológico de no mínimo 700 milhões de anos, entre 1,6 e 0,9 bilhões de anos atrás.

De acordo com F. A. Juncá, L. Funch e W. Rocha (2005), a Chapada Diamantina é constituída por áreas que têm sofrido grande retrabalhamento, gerando um relevo dissecado



Fig. 2 - Área do Vale do Pati-Bahia, Brasil ocupada por Dicranopteris flexuosa.

Fig. 2 - Pati Valley area occupied by Dicranopteris flexuosa. 
com vales profundos, onde a altitude varia de $200 \mathrm{~m}$ a $800 \mathrm{~m}$. O relevo apresenta um aspecto bastante acidentado, com grandes maciços residuais, topos rochosos e encostas íngremes (F. A. Juncá, L. Funch e W. Rocha, 2005).

A área de estudo desta pesquisa (fig. 3) apresenta uma dimensão de aproximadamente 3.393,17 ha, a qual encontra-se inserida nas coordenadas UTM 229875E/8591062N - 240261E/8582322N Zona 24 Sul.

\section{Aquisição e processamento de dados}

Para esta pesquisa, foram utilizadas imagens disponíveis no Banco de Dados do Serviço Geológico do Estados Unidos (USGS), onde foram adquiridas imagens dos anos de 2002, 2006, 2010 e 2014 respectivamente Landsat 7 sensor ETM+, Landsat 5 sensor TM, Landsat 5 sensor TM e Landsat 8 sensor OLI, todas com órbita/ponto 217/69 e resolução espacial de 30 metros.

As imagens supracitadas não correspondem às mesmas épocas dos anos, pois foi levado em conta a menor cobertura de nuvens no local, com o intuito de obter um melhor processamento. Todas as etapas de processamento foram realizadas nas plataformas ENVI 5.1 e ArcGis 10.1 .

A princípio, foi feito os recortes das imagens, delimitando assim a área do Vale, em seguida foi aplicado o método de calibração radiométrica (radiometric calibration) e posteriormente foi realizada a correção atmosférica utilizando o módulo Quick Atmospheric Correction (QUAC).

Uma vez realizadas as etapas de pré-processamento, foram efetuadas as classificações supervisionadas das imagens, onde buscou-se indentificar as classes $D$. flexuosa, Sticherus sp (outra espécie de samambaia também presente no local), vegetação em geral, "gerais", solo, sombra e nuvem, a partir de dois algoritmos diferentes: Redes Neurais e Máxima Verossimilhança, ambos presentes na plataforma ENVI 5.1.

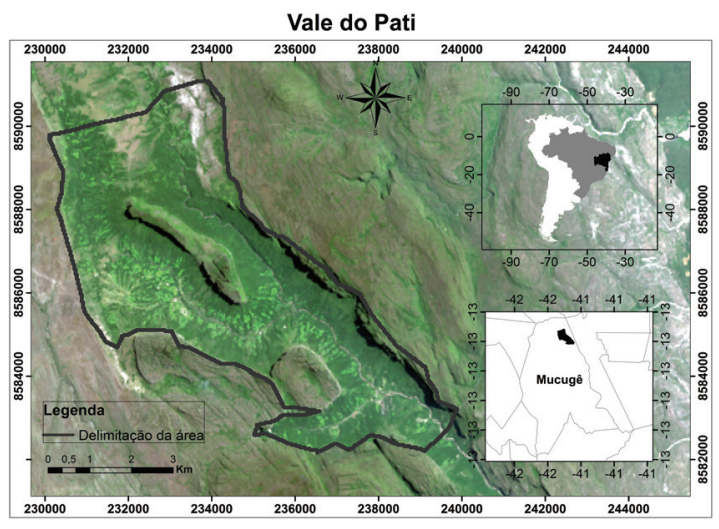

Fig. 3 - Mapa de localização da área de estudo.

Fig. 3 - Location map of study area.
De acordo com A. P. Crósta (1993), a classificação de imagens de satélite é a técnica utilizada para identificar e distinguir diferentes materiais superficiais contidos na mesma. Este processo tem sido muito utilizado em diversas áreas, tendo como principal finalidade a separação de zonas a partir da diferenciação dos padrões espectrais presentes na imagem (M. B. Coutinho, 2010).

De acordo com P. R. Meneses e E. E. Sano (2012), para o sucesso da classificação, é imprescindível o conhecimento dos dados e das classes de objetos que existem na área, antes de se iniciar o processo de classificação. Desta forma, foi realizado um trabalho de campo no vale do Pati, de 2 a 6 de Junho de 2015, com a finalidade de caracterizar a área de estudo, coletando pontos com auxílio do GPS de navegação para verificar áreas com a presença da samambaia. Esses pontos foram utilizados para treinar os algoritmos empregados para a classificação e fazer reconhecimento das classes estabelecidas, georrefenciando estas também com o auxílio do GPS.

O algoritmo Redes Neurais (Redes) foi escolhido pelo fato de vir sendo bastante utilizado por outros pesquisadores e na maioria dos casos ter atingido sucesso nos resultados. Visto que, os produtos alcançados até o momento sugerem que as redes neurais podem ser sólidas quando os dados espectrais são indistintos ou esparsos, e as mesmas são capazes de produzir exatidões que excedem a maioria dos métodos de reconhecimento de padrão que utilizam a estatística convencional (S. R. Yool, 1998). Uma rede neural é constituída por elementos de processamento simples que são denominados como neurônios (M. C. Nelson, W. T. Illingworth, 1991 e S. V. Kartalopoulos, 1996 in W. de J. S da Franca-Rocha, 2015). Esses, fazem operações sobre dados locais, que são as entradas recebidas pelas suas conexões. Dessa forma, o comportamento inteligente de uma Rede Neural é gerado pelas interações entre esses elementos da rede (W. de J. S. da Franca-Rocha, 2015). Segundo W. de J. S. da FrancaRocha (2015) a característica mais importante deste algoritmo é sua habilidade de aprendizado, melhorando cada vez mais o seu desempenho. Ao mesmo tempo, o classificador baseado em Máxima Verossimilhança (MaxVer) também tem se mostrado muito eficiente, assim como o funcionamento das Redes, neste algoritmo deve ser fornecido ao sistema um conjunto de dados ou amostras que representem bem as classes de uso e cobertura da terra para que o classificador tenha condições de definir um esquema de dispersão em torno da média, bem como a distribuição das probabilidades dos pixels pertencerem ou não à determinada classe $(M$. M. França, 2007), ou seja, as classes de treinamento que são utilizadas avaliam a forma da distribuição dos pixels contidos em cada classe no espaço de $n$ bandas, como também a localização do centro de cada classe ( $P$. R. Meneses e E. E. Sano, 2012). 
Para aplicação destas classificações foram coletadas nas imagens amostras representativas das seis classes supracitadas, com base no trabalho de campo realizado. Para o cálculo da matriz de confusão e da acurácia foram gerados dois conjunto de dados, um para a classificação, pelos métodos citados anteriormente, e outro para intuito de comparação pós classificação.

\section{Resultados e discussões}

A partir das classificações supervisionadascom o algoritmo Redes Neurais foram gerados mapas de uso e cobertura do solo (fig. 4) referentes a cada ano, os quais permitiram analisar a proliferação da Dicranopteris flexuosa, assim como da samambaia Sticherus $s p$, na escala temporal estudada e posteriormente quantificar as áreas já povoadas por elas.

$\mathrm{Na}$ TABELA I observa-se os resultados das acurácias obtidas pelas matrizes de confusão, bem como as áreas de ocupação das samambaias em cada ano, a partir deste classificador.

A matriz de confusão gerada a partir das classificações feitas com o algoritmo Redes Neurais, além de apresentar a acurácia, como já exposto anteriormente, descreveu ainda os erros de omissão e comissão como mostra a TABELA II.

Baseado nas classificações supervisionadas por Máxima Verossimilhança também foram gerados mapas de uso e cobertura do solo (fig. 5) de cada ano, e a partir deles quantificou-se a proliferação da $D$. flexuosa bem

TABELA I - Resultado da acurácia e áreas ocupadas pelas samambaias nos anos estudados, de acordo as Redes Neurais.

TABLE I - Accuracy results and measurement of the areas occupied by ferns in the years studied, according to Neural Networks classifier.

\begin{tabular}{|c|c|c|c|c|}
\hline & 2002 & 2006 & 2010 & 2014 \\
\hline Acurácia & $95 \%$ & $97,5 \%$ & $96,9 \%$ & $99,8 \%$ \\
\hline Área D. flexuosa & $397,7 \mathrm{ha}$ & $488,7 \mathrm{ha}$ & $241,7 \mathrm{ha}$ & $321,7 \mathrm{ha}$ \\
\hline Área Stichirus sp & $-\ldots--$ & $\ldots--$ & $97,9 \mathrm{ha}$ & $77 \mathrm{ha}$ \\
\hline
\end{tabular}

como da samambaia Sticherus $s p$, nos anos que se tinha ocorrência da mesma, na escala temporal estudada, como mostra a TABELA III.

A partir do cálculo das áreas ocupadas pelas samambaias foi feita a porcentagem respectiva a essas áreas com relação ao total da área do vale (TABELA IV), de forma a observar com mais facilidade o avanço das mesmas.

A matriz de confusão calculada a partir das classificações com o algoritmo MaxVer, além de apresentar a acurácia, como mostra a TABELA III, descreveu ainda os erros de omissão e comissão como exibe a TABELA V.

Os algoritmos utilizados neste trabalho apresentaram resultados bastante distintos em alguns anos. Foi possível observar inclusive a expressiva diferença quanto às áreas ocupadas pelas espécies, quando comparadas às classificações. Algumas delas mais visíveis que outras, como o ano de 2010, cujo classificador MaxVer apresenta acurácia bem abaixo dos apresentados pelo classificador Redes Neurais. Esse fato pode ser explicado pela confusão que a classificação MaxVer fez entre as classes sombra e

TABELA III - Resultados de acurácia e áreas ocupadas pelas samambaias nos anos estudados com base no algoritmo MaxVer.

TABLE III - Accuracy results and measurement of the areas occupied by ferns in the years studied, according to MaxVer algorithm.

\begin{tabular}{|c|c|c|c|c|}
\hline & 2002 & 2006 & 2010 & 2014 \\
\hline Acurácia & $95,2 \%$ & $94 \%$ & $82,9 \%$ & $91,8 \%$ \\
\hline Área D. flexuosa & $583,4 \mathrm{ha}$ & $948,2 \mathrm{ha}$ & $423,1 \mathrm{ha}$ & $1239 \mathrm{ha}$ \\
\hline Área Sticherus sp & $-\ldots--$ & $\ldots--$ & $476,5 \mathrm{ha}$ & $83,1 \mathrm{ha}$ \\
\hline
\end{tabular}

TABELA IV - Percentagem das áreas ocupadas pelas samambaias no Vale

TABLE IV - Fern occupied area percent in Pati Valley

\begin{tabular}{|c|c|c|c|c|}
\hline & 2002 & 2006 & 2010 & 2014 \\
\hline Área D. flexuosa & $9,8 \%$ & $12,3 \%$ & $6,1 \%$ & $8,1 \%$ \\
\hline Área Sticherus sp & $-\ldots--$ & $-\ldots$ & $2,5 \%$ & $1,9 \%$ \\
\hline
\end{tabular}

TABeLA II - Erros de comissão e omissão nas classificações por Redes Neurais.

TABLE II - Commission and omission errors in the classifications by Neural Networks.

\begin{tabular}{|c|c|c|c|c|}
\hline Classes & $\begin{array}{l}\text { Erro de } \\
\text { comissão/omissão } \\
2002\end{array}$ & $\begin{array}{c}\text { Erro de } \\
\text { comissão/omissão } \\
2006\end{array}$ & $\begin{array}{c}\text { Erro de } \\
\text { comissão/omissão } \\
2010\end{array}$ & $\begin{array}{c}\text { Erro de } \\
\text { comissão/omissão } \\
2014\end{array}$ \\
\hline Sombra & $0 \% / 0 \%$ & $0 \% / 0 \%$ & $1,2 \% / 0 \%$ & $0 \% / 0 \%$ \\
\hline Gerais & $15,5 \% / 13 \%$ & $13,2 \% / 11,5 \%$ & $4,9 \% / 1,3 \%$ & $0 \% / 0 \%$ \\
\hline Nuvem & $0 \% / 1,4 \%$ & $\cdots$ & $0 \% / 0 \%$ & $\cdots$ \\
\hline Solo exposto & $19,1 \% / 22,5 \%$ & $6,6 \% / 7,6$ & $12,5 \% / 8,8 \%$ & $0 \% / 0 \%$ \\
\hline D. flexuosa & $11,1 \%$ / 0\% & $3 \% / 0 \%$ & $9,1 \% / 11,8 \%$ & $0 \% / 1,5 \%$ \\
\hline Sticherus sp & $\cdots-\cdot$ & $\cdots-\cdot$ & $0 \%$ / 27,3\% & $0 \% / 0 \%$ \\
\hline Vegetação em geral & $0,6 \% / 2,5 \%$ & $0 \% / 0,5 \%$ & $5,7 \% / 5,7 \%$ & $0,5 \% / 0 \%$ \\
\hline
\end{tabular}


RISCOS - Associação Portuguesa de Riscos, Prevenção e Segurança

TABELA V - Erros de comissão e omissão ocorridos nas classificações por Máxima Verossimilhança.

$T_{A B L E} V$ - Commission and omission errors in the classifications by MaxVer.

\begin{tabular}{|c|c|c|c|c|}
\hline Classes & $\begin{array}{c}\text { Erro de } \\
\text { comissão/omissão } \\
2002\end{array}$ & $\begin{array}{c}\text { Erro de } \\
\text { comissão/omissão } \\
2006\end{array}$ & $\begin{array}{c}\text { Erro de } \\
\text { comissão/omissão } \\
2010\end{array}$ & $\begin{array}{c}\text { Erro de } \\
\text { comissão/omissão } \\
2014\end{array}$ \\
\hline Sombra & $0 \% / 0,4 \%$ & $0 \% / 0 \%$ & $0 \% / 33,8 \%$ & $0 \% / 2,8 \%$ \\
\hline Gerais & $4,4 \% / 13 \%$ & $10 \% / 13,5 \%$ & $62,1 \% / 0 \%$ & $15,9 \% / 18,1 \%$ \\
\hline Nuvem & $0 \% / 0 \%$ & $\cdots--\cdot$ & $0 \% / 0 \%$ & $\cdots-\cdot$ \\
\hline Solo exposto & $20,2 \% / 5,6 \%$ & $7,5 \% / 5,4 \%$ & $0 \% / 8,7 \%$ & $46,7 \% / 25 \%$ \\
\hline D. Flexuosa & $22,8 \% / 15,6 \%$ & $40,7 \% / 0 \%$ & $10 \% / 5,3 \%$ & $3 \% / 0 \%$ \\
\hline Sticherus $s p$ & $\cdots-\cdots$ & $\cdots+\cdots$ & $15,4 \% / 21,4 \%$ & $0 \% / 5,9 \%$ \\
\hline Vegetação em geral & $2,6 \% / 6,1 \%$ & $0 \% / 10,7 \%$ & $1,2 \% / 2,3 \%$ & $0,5 \% / 5,2 \%$ \\
\hline
\end{tabular}
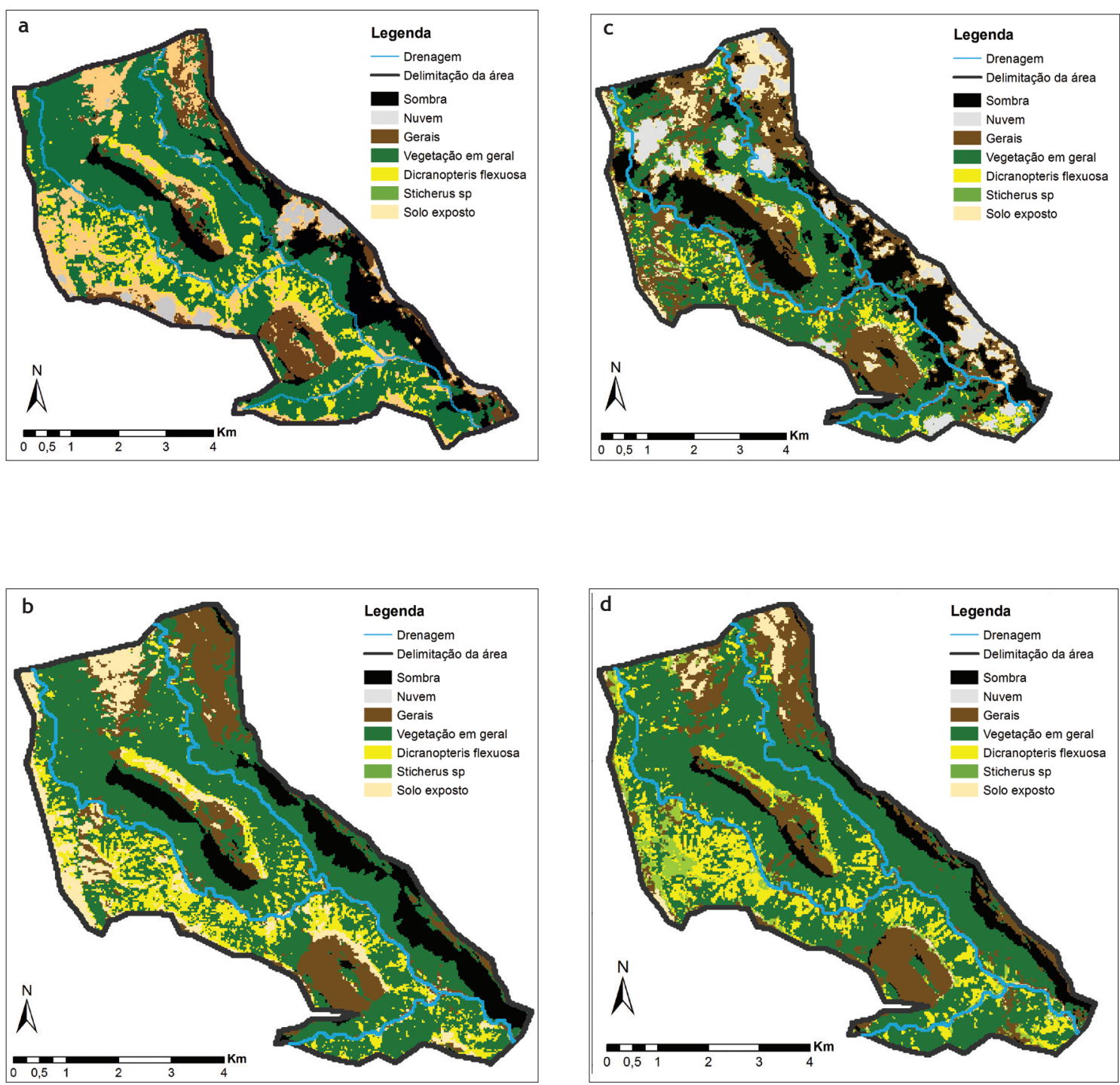

Fig. 4 - Mapa de uso e cobertura do solo, a partir do classificador Redes Neurais: a) 2002; b) 2006; c) 2010; d) 2014.

Fig. 4 - Land Use/Land Cover Map using Neural Networks classifier: a) 2002; b) 2006; c) 2010; d) 2014. 
gerais, de forma que somente $66,1 \%$ da classe sombra foi classificada corretamente enquanto $33,9 \%$ foi confundida com a classe gerais, bem como $21,4 \%$ da classe Sticherus sp foi confundida com a classe $D$. flexuosa.

Ao analisar a disseminação das espécies através dos anos investigados, no Vale do Pati, é possível perceber também uma drástica redução das áreas ocupadas por elas entre os anos 2006 e 2010, fato que se explica, provavelmente, em razão das grandes ocorrências de incêndios que aconteceram no ano de 2008 em todo o Parque Nacional da Chapada Diamantina, totalizando, de acordo com dados do INPE in T. B. Silva (2013), 243 focos de calor somente naquele ano.

É necessário ressaltar que as áreas apresentadas neste artigo, sofreram certo equívoco, visto que não foi possível identificar pixels correspondentes à drenagem,
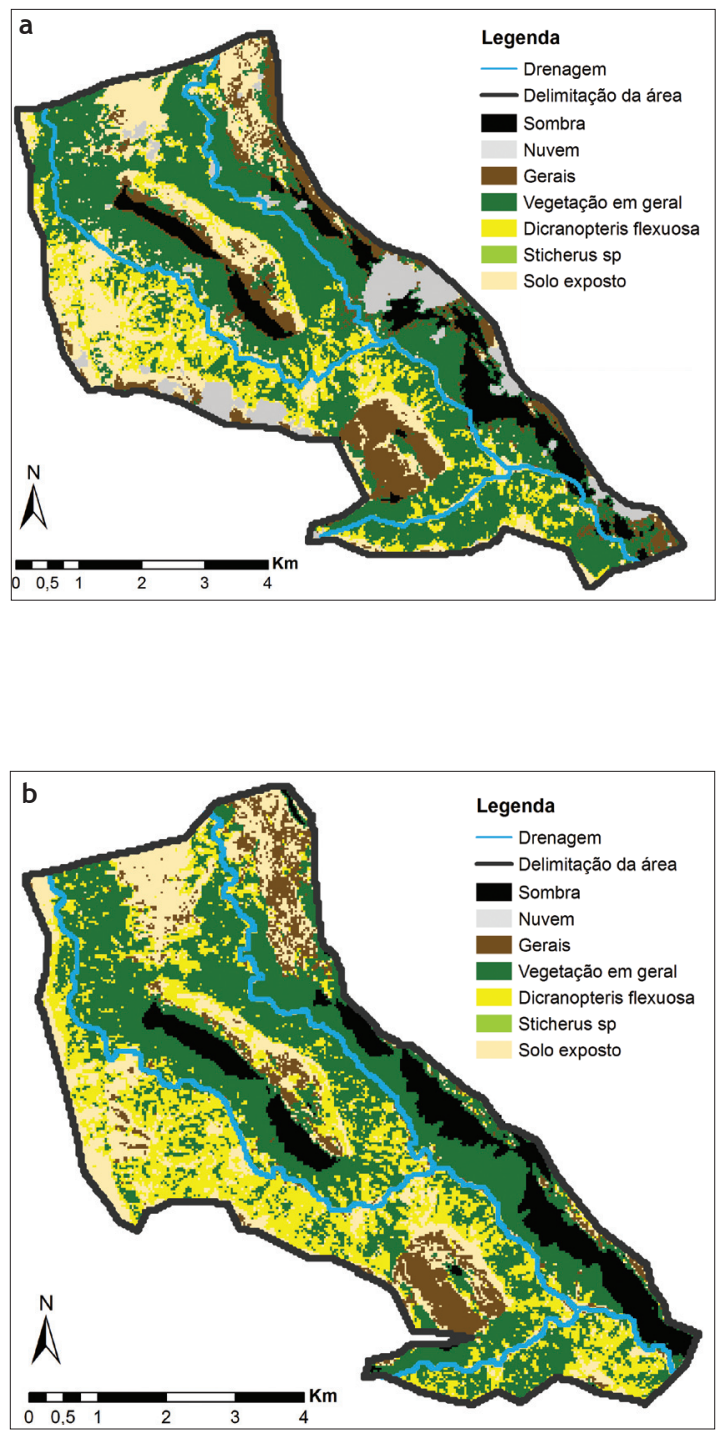

devido à baixa resolução da imagem, o que fez com que algumas classes fossem classificadas como a mesma.

Abordando um estudo comparativo entre as técnicas de classificação por Máxima Verossimilhança e Redes Neurais, assim como R. B. Queiroz, A. G. Rodrigues e A. T. Gómez (2004) o fizeram, conclui-se que o classificador Redes Neurais revela ser mais adequado para classificar imagens de satélite, tendo em vista que o mesmo se mostrou mais eficaz quando comparado ao classificador MaxVer.

C. Gelelete e C. Volotão (2007) também realizaram um estudo utilizando o algoritmo Redes Neurais onde, segundo eles, houve menor ocorrência de confusão entre classes, apesar de algumas sombras terem sido classificadas como água. Dessa forma, atesta-se a potencialidade deste classificador em identificar as classes com boa precisão, superando resultados obtidos com o classificador de Máxima Verossimilhança.

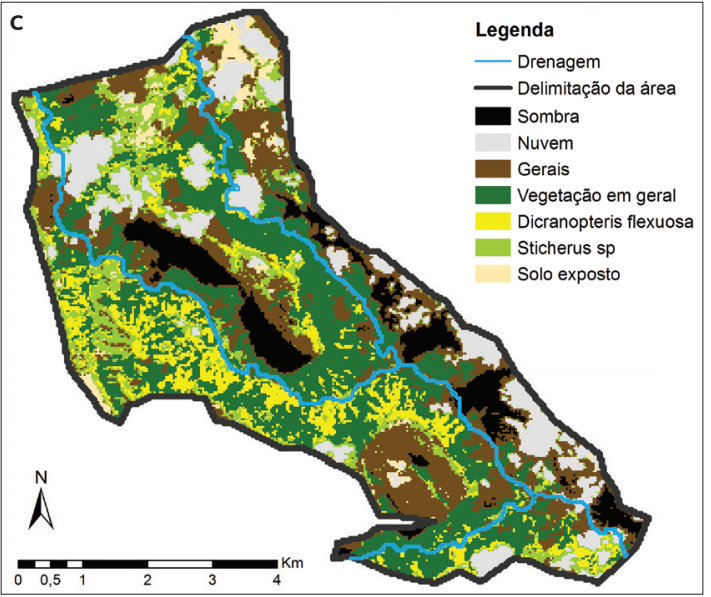

Fig. 5 - Mapa de uso e cobertura do solo, baseado no classificador Máxima Verossimilhança: a) 2002; b) 2006; c) 2010 ; d) 2014.

Fig. 5 - Land Use/Land Cover Map, using Maximum Likelihood classifier: a) 2002; b) 2006; c) 2010 ; d) 2014. 
M. M. França, E. I. F. Filho e B. T. De L. Xavier em 2009, apresentaram uma pesquisa fazendo análise do uso da terra de Viçosa-MG, Brasil, a partir de classificações supervisionadas com os algoritmos Redes Neurais e Máxima Verossimilhança, bem como o presente trabalho. Nesta pesquisa, os autores constataram que o classificador Redes Neurais, apesar de exibir índices Kappa ligeiramente menores que os apresentados pelo classificador MaxVer, apresentou menor ocorrência de confusão entre pixels internos por classes, comprovando índices de exatidão mais significativos. Estes pesquisadores concluíram ainda que uma hipótese plausível para essa ocorrência consistiu na utilização do algoritmo backpropagation pelas Redes. Este, ao treinar os pixels ajusta os pesos de forma a conseguir adaptar os diferentes estímulos de entrada, verificando-se dessa forma, o aprendizado.

Em 2001, S. R. A. Ribeiro e J. S. Centeno, também utilizaram dos algoritmos Redes Neurais e Máxima Verossimilhança para fazer um estudo de classificação de uso do solo. Os autores apresentaram como resultados que o algoritmo Redes Neurais em todos os exemplos foi superior ao algoritmo MaxVer, salientando que este último pode ser melhorado utilizando-se um maior número de amostras de treinamento, pois a título de comparação foi utilizada a mesma quantidade de amostras para ambos os classificadores, notando-se dessa forma, que as Redes Neurais requerem menor número de amostras para apresentarem bom desempenho de classificação.

Em contrapartida, C. S. Chagas, C. A. O. Vieira, E. I. Fernandes Filho e W. de C. Júnior (2008) apresentaram um estudo comparativo entre os algoritmos Redes e MaxVer na classificação de níveis de pastagem, avaliando de forma diferente esta comparação. Segundo Kanellopoulos e Wilkinson (1997) in C. S. Chagas, C. A. O. Vieira, E. I. Fernandes Filho e W. de C. Júnior (2008), nos vários estudos abordando este tipo de comparação tem-se negligenciado a existência de diferenças significativas entre o desempenho desses classificadores, pois quando se consideram as classes, individualmente, o classificador Redes pode não ser tão eficiente quanto aparenta. Dessa forma, os autores observaram nesta pesquisa que a maior exatidão obtida individualmente foi na classe café, pelo algoritmo MaxVer, constatando-se $100 \%$ de exatidão, enquanto o classificador Redes apresentou 96\%. Por conseguinte, embora os resultados das Redes apresentem desempenho superior em relação aos valores absolutos, o classificador MaxVer não se distancia muito deste bom desempenho.

\section{Conclusão}

Ao fim desta pesquisa observou-se que a metodologia empregada na mesma mostrou-se bastante eficaz. Posto que a partir das classificações desenvolvidas foi possível mapear as classes de uso e cobertura da terra do Vale do Pati, por meio de Sensoriamento Remoto no período entre os anos de 2002 a 2014, bem como também identificar na imagem a distribuição espacial da espécie invasora Dicranopteres flexuosa no recorte temporal escolhido.

Baseado nas classificações geradas a partir dos diferentes algoritmos utilizados, quantificou-se as mudanças no padrão de uso e ocupação da terra e na distribuição espacial da espécie estudada, ao longo do período analisado com precisão, abordando assim suas áreas de ocorrência, podendo dessa forma contribuir para o manejo adequado do Vale do Pati.

Com suporte na comparação realizada entre os algoritmos de classificação observou-se ainda que o algoritmo Redes Neurais foi mais eficiente do que o Máxima Verossimilhança, pois apresentou em suma menor ocorrência de confusão e índices de exatidão maiores.

\section{Referências bibliográficas}

Borges, V. Costa. e Lobão, J. S. Britto (2014). Mapeamento do uso da terra na comunidade tradicional do Vale do Pati/Parque Nacional da Chapada Diamantina-Ba. In: Anais do VI Congresso Iberoamericano de Estudios Territoriales y Ambientales. São Paulo, p. 5072- 5089.

Cezar, R. Valle. (2011). Carta Geoambiental da região turística do Vale do Pati- Chapada Diamantina, $\mathrm{Ba}$ (Mestrado em Geociências e Meio Ambiente). Instituto de Geociências e Ciências Exatas, Universidade Estadual Paulista, Rio Claro- SP.

Chagas, C. S., Vieira, C. A. O., Filho, E. I. F e Júnior, W. de C. (2008). Utilização de Redes Neurais artificiais na classificação de níveis de degradação em pastagens. Revista Brasileira de Engenharia Agrícola e Ambiental. Campina Grande, v. 13, n. 3, p. 319 327. Maio-Out.

Coutinho, M. B. (2010). Mapeamento Geológico Multiespectral na Bacia Carbonática de Una-Utinga-Ba e das mineralizações de $\mathrm{Pb}$ associadas (Monografia , Bacharel em Geologia). Instituto de Geociências, Universidade Federal da Bahia, Salvador, 58 p.

Crósta, A. P. (1993). Processamento digital de imagens de sensoriamento remoto. Instituto de Geociências, Univ. Estadual de Campinas, SP.

Franca-Rocha, W. de J. Sant'Anna da. (2015). Modelagem metalogenética espacial: estudo de caso na Bacia de Irecê. Salvador: CBPM.

França, M. M. (2007). Avaliação de Classificações Supervisionadas Com Redes Neurais Artificiais MAXVER para Caracterização do Uso da Terra no Município de Viçosa-MG (Monografia em Geografia). Departamento de Artes e Humanidades da Universidade Federal de Viçosa, Viçosa-MG. 
França, M. M., Filho, E. I. F. e Xavier, B. T. De L. (2009). Análise do uso da terra no município de Viçosa-MG mediado por classificações supervisionadas com Redes Neurais artificiais e MaxVer. Revista Brasileira de Geografia Física. Recife, v. 2, n. 03, p. 92-101, Set-Dez.

Funk, J. L. (2013). The physiology of invasive plants in low-resource environments. Conservation physiology. v. 1.

Gelelete, Cláudio e Valetão, C. F. de Sá (2007). Análise do potencial de classificação do uso e cobertura do solo por meio de rede neural. In: Anais do XII Simpósio Brasileiro de Sensoriamento Remoto. Florianópolis, p. 5779-5785.

Hojo-Souza, N. Satchiko, Carneiro, C. Martins e Santos, R. Cardoso (2010). Pteridium aquilinum: O QUE SABEMOS E O QUE AINDA FALTA SABER. Biosci. J., Uberlândia, v. 26, n. 5, p. 798-808, Sept./Oct.

Jensen, John. R. (2009). Sensoriamento Remoto do Ambiente: uma perspectiva em recursos terrestres. 2 ed. São José dos Campos, SP: Parênteses.

Juncá, F. Acunã, Funch, L. e Rocha, W. (org.) (2005). Biodiversidade e Conservação da Chapada Diamantina. Brasília: Ministério do Meio Ambiente.

Lehmann, D. R. Marques (2008). Estudos sobre a propagação de Gleichenella pectinata (Willd.) Ching (Pteridófita Gleicheniaceae) (Dissertação (Mestrado em Biologia Vegetal). Universidade Federal de Santa Catarina, Florianópolis, $44 \mathrm{p}$.

Meneses, P. Roberto e Sano, E. Eyjin (2012). Classificação pixel a pixel de imagem. In: Meneses, P. Roberto e Almeida, Tati. Introdução ao Processamento de Imagens de Sensoriamento Remoto. Unb: Brasília. Cap. 12.

MMA - MINISTÉRIO DO MEIO AMBIENTE (2007). Plano de Manejo para o Parque Nacional da Chapada Diamantina. Brasília.

Nonato, F. Regina. (2005) Pteridófitas. In: Juncá, F. Acunã, Funch, L. e Rocha, W. (org.). Biodiversidade e Conservação da Chapada Diamantina. Brasília: Ministério do Meio Ambiente.
Pedreira, Augusto J. (1997). Sistemas Deposicionais da Chapada Diamantina Centro-Oriental, Bahia. Revista Brasileira de Geociências, Vol-27 (3), p. 229-240.

Queiroz, R. Baptista, Rodrigues, A. Gabriel e Gómez, A. Tórgo (2004). Estudos comparativos entre técnicas Máxima Verossimilhança Gaussiana e Redes Neurais na classificação de imagens IR-MSS CBERS 1. In: II Workshop de Tecnologia da Informação Aplicada ao Meio Ambiente-CBcomp.

Queiroz, M. Hering (1994). Approche phytoécologique et dynamique des formations végétales secondaires développées après abandon des activités agricoles, dans le domaine de la Forêt Ombrophile dense de versant (Forêt Atlantique) à Santa Catarina Brésil (Thèse de Doctorat). École Nationale du Génie Rural des Eaux et de Forêts. Nancy, França.

Ribeiro, S. R. A. e Centeno, J. S. (2001). Classificação do uso do solo utilizando Redes Neurais e o algoritmo MaxVer. In: Anais do X Simpósio Brasileiro de Sensoriamento Remoto, Foz do Iguaçu, p. 1341-1348.

Seabra, G. de Farias. (1998). Do garimpo aos ecos do turismo: o Parque Nacional da Chapada Diamantina (Tese de doutorado). Departamento de geografia da USP, São Paulo.

Silva, T. Bonfim (2013). Quantificação e análise espacial dos focos de calor no Parque Nacional da Chapada Diamantina - BA. In: Anais do XVI SIMPÓSIO BRASILEIRO DE SENSORIAMENTO REMOTO. FOZ do Iguaçu, p. 6969-6976.

Tryon, R. Milton e Tryon, A. Faber (1982). Dicksoniaceae. In R. Tryon e A. F. Tryon (Eds.) Fern and allied plants with special reference to Tropical America SpringerVerlag. New York, EUA. 138-154.

Yool, Stephen R. (1998). Land cover classification in rugged areas using simulated moderate-resolution remote sensor data and an artificial neural network. International Journal of Remote Sensing, v.19, p.85-96. 